\title{
Erratum to: International workshop on insecticide resistance in vectors of arboviruses, December 2016, Rio de Janeiro, Brazil
}

\author{
Vincent Corbel ${ }^{1 *}$, Dina M. Fonseca², David Weetman ${ }^{3}$, João Pinto ${ }^{4}$, Nicole L. Achee ${ }^{5}$, Fabrice Chandre ${ }^{1}$, \\ Mamadou B. Coulibaly ${ }^{6}$, Isabelle Dusfour ${ }^{7}$, John Grieco ${ }^{5}$, Waraporn Juntarajumnong ${ }^{8}$, Audrey Lenhart ${ }^{9}$, \\ Ademir J. Martins ${ }^{10}$, Catherine Moyes ${ }^{11}$, Lee Ching Ng ${ }^{12}$, Kamaraju Raghavendra ${ }^{13}$, Hassan Vatandoost ${ }^{14}$, \\ John Vontas ${ }^{15,16}$, Pie Muller ${ }^{17}$, Shinji Kasai ${ }^{18}$, Florence Fouque ${ }^{19}$, Raman Velayudhan ${ }^{20}$, Claire Durot ${ }^{1}$ \\ and Jean-Philippe David ${ }^{21,22^{*}}$
}

\section{Erratum}

After the publication of the article [1], it was realised that the attribution text for Fig. 3 was not correct. The corrected Fig. 3 attribution is included below.

A corrected version of Fig. 3 is included with this Erratum.

\footnotetext{
Author details

'Institut de Recherche pour le Développement (IRD), Maladies Infectieuses et Vecteurs, Ecologie, Génétique, Evolution et Contrôle (MIVEGEC, UM1-CNRS 5290-IRD 224), B.P. 64501, 911 Avenue Agropolis, 34394 Montpellier Cedex 5, France. ${ }^{2}$ Center for Vector Biology, Rutgers University (RU), 180 Jones Avenue, New Brunswick, NJ 08901, USA. ${ }^{3}$ Department of Vector Biology, Liverpool School of Tropical Medicine (LSTM), Pembroke Place, Liverpool L35QA, UK. ${ }^{4}$ Global Health and Tropical Medicine, GHTM, Instituto de Higiene e Medicina Tropical, IHMT, Universidade Nova de Lisboa, UNL, Rua da Junqueira 100, 1349-008 Lisbon, Portugal. ${ }^{5}$ Department of Biological Sciences, University of Notre Dame (UND), Eck Institute for Global Health, 239 Galvin Life Science Center, Notre Dame, Indiana 46556, USA. 'Malaria Research and Training Center (MRTC), Point G, Bamako B.P. 1805, Mali. ${ }^{7}$ Institut Pasteur de la Guyane (IPG), 23 avenue Pasteur B.P. 6010, 97306 Cayenne Cedex, French Guiana. ${ }^{8}$ Department of Entomology, Kasetsart University (KU), 50 Ngam Wong Wan Rd, Ladyaow, Bangkok, Chatuchak 10900, Thailand. ${ }^{9}$ Center for Global Health/Division of Parasitic Diseases and Malaria/Entomology Branch, U.S. Centers for Disease Control and Prevention (CDC), 1600 Clifton Rd. NE, MS G-49; Bldg. 23, Atlanta, GA 30329, USA.

${ }^{10}$ Instituto Oswaldo Cruz (Fiocruz), Avenida Brasil 4365, Rio de Janeiro/RJ CEP, Manguinhos 21040-360, Brazil. " Big Data Institute, Li Ka Shing Centre for Health Information and Discovery, University of Oxford, Oxford OX3 7LF, UK.

*Correspondence: vincent.corbel@ird.fr; jean-philippe.david@univ-grenoblealpes.fr

'Institut de Recherche pour le Développement (IRD), Maladies Infectieuses et Vecteurs, Ecologie, Génétique, Evolution et Contrôle (MIVEGEC, UM1-CNRS 5290-IRD 224), B.P. 64501, 911 Avenue Agropolis, 34394 Montpellier Cedex 5, France

${ }^{21}$ Centre National de la Recherche Scientifique (CNRS), Laboratoire d'Ecologie Alpine (LECA), UMR 5553 CNRS, 2233 rue de la piscine, 38041 Grenoble Cedex 9, France
}

${ }^{12}$ Environmental Health Institute (EHI), National Environment Agency (NEA), 11 Biopolis Way, Helios Block, \#04-03/04 \& \#06-05, /08 Singapore, Republic of Singapore. ${ }^{13}$ Department of Health Research, National Institute of Malaria Research (NIMR), Gol Sector 8, Dwarka, Delhi 110 077, India. ${ }^{14}$ Department of Medical Entomology \& Vector Control, School of Public Health and Institute for Environmental Research, Tehran University of Medical Sciences (TUMS), Pour Sina Street, P.O. Box: 14155-6446, Tehran, Iran. ${ }^{15}$ Institute Molecular Biology and Biotechnology (IMBB), Foundation for Research and Technology (FORTH), Panepistimioupoli, Voutes, 70013 Heraklio, Crete, Greece. ${ }^{16}$ Pesticide Science Laboratory, Agricultural University of Athens, leara Odoes 75, 118 Athens, Greece. ${ }^{17}$ Department of Epidemiology and Public Health, Swiss Tropical and Public Health Institute, Socinstrasse 57, PO Box, 4002, Basel, Switzerland. ${ }^{18}$ Department of Medical Entomology, National Institute of Infectious Diseases, 1-23-1 Toyama, Shinjukuku, Tokyo, Japan. ${ }^{19}$ Vector Environment and Society Unit, The Special Programme for Research and Training in Tropical Diseases World Health Organization, 20, avenue Appia, CH-1211 Geneva 27, Switzerland. ${ }^{20}$ Vector Ecology and Management, Department of Control of Neglected Tropical Diseases (HTM/NTD), World Health Organization, 20 Avenue Appia, CH-1211 Geneva 27, Switzerland. ${ }^{21}$ Centre National de la Recherche Scientifique (CNRS), Laboratoire d'Ecologie Alpine (LECA), UMR 5553 CNRS, 2233 rue de la piscine, 38041 Grenoble Cedex 9, France. ${ }^{22}$ Université Grenoble-Alpes, Domaine universitaire de Saint-Martin d'Hères, 2233 rue de la piscine, 38041 Grenoble Cedex 9, France.

Received: 8 August 2017 Accepted: 8 August 2017

Published online: 21 August 2017

\section{References}

1. Corbel V, Fonseca DM, Weetman D, Pinto J, Achee NL, Chandre F, et al. International workshop on insecticide resistance in vectors of arboviruses, December 2016, Rio de Janeiro, Brazil. Parasit Vectors. 2017;10:278. doi:10. 1186/s13071-017-2224-3.

2. Kraemer MU, Sinka ME, Duda KA, Mylne AQ, Shearer FM, Barker CM, et al. The global distribution of the arbovirus vectors Aedes aegypti and Ae. albopictus. elife. 2015;4:e08347. 


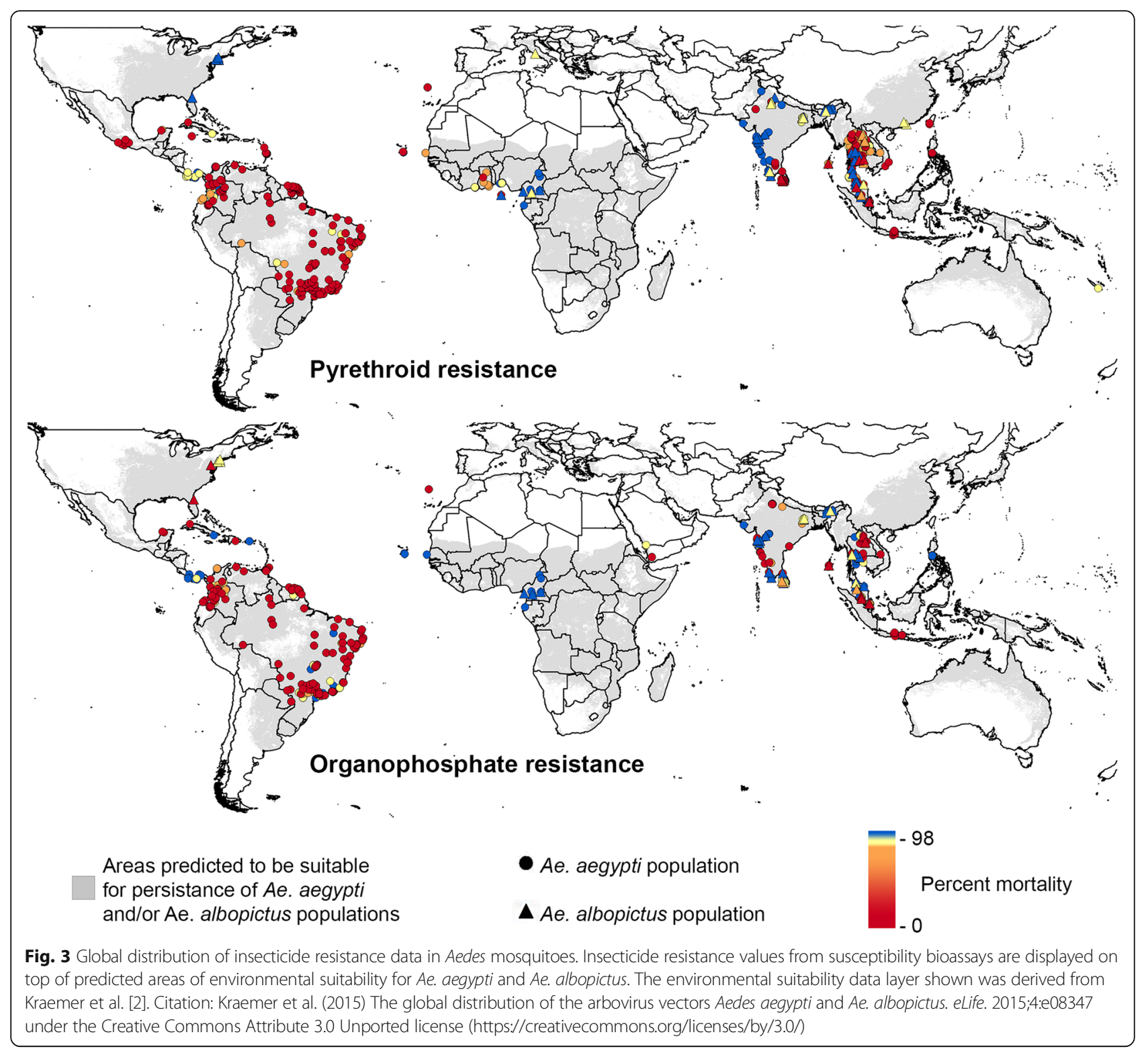

